\title{
DiE WEIBLICHE LEICHE BEI DOSTOEVSKIJ
}

Slata Kozakova, München, Deutschland, slata.kozakova@Irz.unimuenchen.de

DOI: 10.31902/fll.38.2021.11

In diesem Artikel wird das Konzept der „schönen weiblichen Leiche“ nach E. Bronfen auf F. M. Dostoevskijs „Idiot“ (1868) und „Krotkaja“ („Die Sanfte“, 1876) in Hinblick auf die narrative Funktion der toten Frauen angewendet.

Schlüsselwörter: Weiblichkeit, Tod, Leiche, Idiot, Nastas'ja Filippovna, Bronfen.

Genau zwei Texte von Dostoevskij basieren strukturell auf einer weiblichen Leiche, der Roman "Idiot" (1868) und die in der Novemberausgabe von „Dnevnik pisatelja“ („Tagebuch eines Schriftstellers“) erschienene Erzählung „Krotkaja“ („Die Sanfte“, 1876). ${ }^{1}$ Bei der Betrachtung dieser Texte soll hier die Arbeit von Elisabeth Bronfen („Nur über ihre Leiche. Tod, Weiblichkeit und Ästhetik“) genutzt werden, die im Original 1992 als „Over Her Dead Body: Death, Femininity and the Aesthetic" erschienen ist und eine Reihe an künstlerischen Werken analysiert, die den Tod einer schönen Frau zum Thema haben. Sie erklärt diesen Topos, grob gesagt, mit dem Zusammenfallen des Todes, der sich jeder sprachlich-symbolischen Ordnung entzieht, und der Weiblichkeit, die kulturell ebenfalls als ein unfassbares Anderssein kodiert ist. Ausgehend von der Annahme, dass jede literarische Darstellung des Todes ein höchst kunstvoller Akt ist, der eine fehlende Referentialität und die Erinnerung an die Sterblichkeit des Betrachters umspielt, soll hier der Frage nachgegangen werden, wozu und warum auf gerade die Weise Nastas'ja Filippovna und Krotkaja sterben.

${ }^{1}$ Die „winzige, dürre Alte“ („крошечная, сухая старушонка“, Dostoevskij T. 6, 8), deren ekelerregender Tod in "Prestuplenie i nakazanie“ („Verbrechen und Strafe“, 1866) die Grenze markiert, die von Raskol'nikov übertreten wird, stellt einen einmaligen, symbolischen Anlass für alles Folgende dar. Ihre hässliche Leiche demonstriert die blutige Tat; die Spannung des Sujets wird nicht von der Leiche als Rätsel selbst oder ihrer erzähltechnischen Bedeutung, sondern von der prinzipiellen Frage nach der Rechtmäßigkeit des Mordes getragen. 
Wer ist Nastas'ja Filippovna? - Fürst Myškin erfährt von ihr im Zugwaggon, auf dem Weg nach Russland; bereits auf den ersten Seiten bildet sich ein Beziehungsdreieck zwischen dem Fürsten, Rogožin und der geheimnisvollen Frau heraus, das bis ans Ende des Romans seine Spannung hält, bis alle drei sich endgültig voneinander trennen. Rogožin erzählt über Nastas'ja Filippovna, die er von Weitem gesehen und über die er wiederum Auskünfte von einem Bekannten bezogen hat. Rogožin ist wie verhext; er präsentiert Nastas'ja Brillantohrringe, die er vom Geld seines Vaters heimlich gekauft hat, wird von diesem eingesperrt und körperlich „belehrt", flieht von Zuhause, betrinkt sich, wacht im Fieberrausch auf einer Straße auf (Dostoevskij T. 8, 11ff.). Mit einem riesigen Kapital, das er plötzlich von seinem Vater erbt, kehrt Rogožin in die Stadt zurück, in der Hoffnung, der Frau, die einen derart gewaltigen Eindruck auf ihn gemacht hat, nun alles bieten zu können.

Als der Fürst zum ersten Mal das Haus der Epančiny aufsucht, lernt er Nastas'ja Filippovna über ein Porträt kennen:

„-Так это Настасья Филипповна? - промолвил он, внимательно и любопытно поглядев на портрет. - Удивительно хороша! прибавил он тотчас же с жаром. На портрете была изображена действительно необыкновенной красоты женщина. Она была сфотографирована в черном шелковом платье, чрезвычайно простого и изящного фасона; волосы, по-видимому темнорусые, были убраны просто, по-домашнему; глаза темные, глубокие, лоб задумчивый; выражение лица страстное и как бы высокомерное. Она была несколько худа лицом, может быть, и бледна..." $(27)^{2}$

Fürst Myškin ist von diesem Bild fasziniert: „Удивительное лицо! (...) и я уверен, что судьба ее не из обыкновенных. Лицо веселое, а она ведь ужасно страдала, а?“ (31) Dieses Porträt verbindet alle

2 ,'Das ist also Nastassja Filippowna?' fragte er, wobei er das Portrait mit einem aufmerksamen und neugierigen Blick betrachtete. ,Sie ist ja unglaublich schön!' rief er im gleichen Atemzug begeistert aus. Es war das Portrait einer in der Tat ungewöhnlich schönen Frau. Sie hatte sich in einem schwarzen Seidenkleid von außerordentlich einfachem und elegantem Schnitt photographieren lassen; das Haar, dem Anschein nach dunkelblond, war ganz schlicht aufgesteckt; die Augen waren dunkel, tief, die Stirn nachdenklich; der Ausdruck des Gesichts leidenschaftlich und irgendwie hochmütig. Es war ein wenig mager, vielleicht auch blaß..." (Dostojewskij 2016, 45)

3 ,',Ein erstaunliches Gesicht!' antwortete der Fürst, ,und ich bin überzeugt, daß ihr Schicksal ungewöhnlich ist. Das Gesicht ist heiter, aber sie muß doch entsetzlich gelitten haben, nicht wahr?““ (53) 
wichtigen Figuren des Romans - Ganja, der Nastas'ja Filippovna heiraten soll, mit Tockij, der sie loswerden und eine Generalstochter heiraten will, mit dem General Epančin selbst, dem sie von Ganja überlassen werden soll - und wandert von Hand zu Hand, von Betrachter zu Betrachter in der Familie der Epančiny und der Ivolginy weiter. Dann erscheint plötzlich Nastas'ja Filippovna selbst - ein Rätsel, eine Maskerade, bei der immer das Entgegengesetzte von dem durchscheint, was sie zu sein vorgibt. Sie überrascht mit unvorhersehbarem Ton, mit chaotischen Sprüngen zwischen extremen Gesten, zwischen Hass- und Liebesausdrücken, wird mal als "Verrückte" („Женщина сумасшедшая!“, 148), mal als „ungeschliffener Diamant“ („Нешлифованный алмаз“, 149) bezeichnet:

„(...) про Настасью Филипповну установилась странная слава: о красоте ее знали все, но и только; никто не мог ничем похвалиться, никто не мог ничего рассказать." (39) 4 Ihre einzige stabile Eigenschaft bleibt ihre Unbestimmtheit sowie eine unerträgliche, paradoxale Schönheit, die im Porträt zum Höhepunkt kommt: „Эта ослепляющая красота была даже невыносима, красота бледного лица, чуть не впалых щек и горевших глаз; странная красота!“ $(68)^{5}$

Der bevorstehende Tod Nastas'ja Filippovnas steht von Anfang an fest. Kaum ist der Fürst in Sankt Petersburg angekommen, weiß er, dass Rogožin seine Braut (mit dem Nachnamen Baraškova, "barašek" = Böcklein, Lamm) nach der Hochzeit bestimmt abstechen, geradezu schlachten werde. Kurz vor ihrer Trauung mit Rogožin bemerkt Nastas'ja Filippovna über das eigene Spiegelbild, dass es „blass wie ein Toter" sei („бледна как мертвец“, 492). Sie ist auch fast eine Tote, eine zur Todesstrafe Verurteilte, die um ihren festgelegten, fremdbestimmten Tod weiß und ihn kaum hinauszögern kann; ihr Bild fällt mit einem anderen, imaginierten Porträt zusammen, von dem der Fürst so begeistert spricht:

„(...) нарисовать лицо приговоренного за минуту до удара гильотины, когда еще он на эшафоте стоит, перед тем как ложиться на эту доску. (...) Нарисуйте эшафот так, чтобы видна

${ }^{4}$ „SChließlich erfreute sich Nastassja Filippowna eines eigentümlichen Rufs: Ihre Schönheit wurde allgemein gerühmt, aber das war auch alles; niemand durfte sich mit einem Erfolg brüsten, niemand ihr etwas nachsagen." (67)

5 „Diese blendende Schönheit war sogar unerträglich, die Schönheit der Blässe, der leicht eingefallenen Wangen, der brennenden Augen; eine eigentümliche Schönheit!“ (117f.) 
была ясно и близко одна только последняя ступень; преступник вступил на нее: голова, лицо бледное как бумага, священник протягивает крест, тот с жадностию протягивает свои синие губы, и глядит, и - всё знает." (54ff.)

Anders kann Nastas'ja Filippova auch nicht sein: „В вас всё совершенство... даже то, что вы худы и бледны... вас и не желаешь представить иначе..." $(118)^{7}$. Sie ist von Tod gezeichnet und verschmilzt mit dem Tod, zwei Rätsel vereinen sich zu einem Ganzen.

Repräsentationen, so Bronfen, beruhen auf dem Fehlen eines referentiellen Objektes: „In gewissem Sinn ist jedes Bild der Tod des repräsentierten Objekts, denn es bezeichnet, zumindest implizit, daß etwas nicht als real, sondern als Bild gedacht oder erkannt wurde." (Bronfen 45) Das fotografische Porträt ist keine Verdoppelung Nastas'ja Filippovnas, sondern eine Kodierung, die das Original selbst ersetzt. Die Fotografie bewirkt einen geschlossenen, einprägsamen Eindruck, der beim realen (real-fiktionalen) Erscheinen des Originals fehlt, das unfassbar, in seinen Aussagen und Versprechen widersprüchlich bleibt und provokante Gesten mit innigen Geständnissen vereint; Nastas'ja Filippova scheint alles und nichts zugleich zu sein.

Die gefährliche, trügerische Femme fatale bringt Männer um den Verstand, jagt ihnen Angst ein, schürt Begehren, lässt sie ihren sozialen Status vergessen, zieht ein Konglomerat von Verehrern verschiedenster Stellung, Abstammung und Einkommensverhältnisse an. Mal ist sie eine gebildete Salondame, mal Rogožins „Königin“, die durch Prunk und exzentrische Ausfälle auf sich aufmerksam macht und mindestens vier geplante Verlobungen in ihrem Umkreis aufgehen lässt. Dieses Karnevaleske im Sinne Michail Bachtins (207f.) bekommt am deutlichsten General Epančin zu spüren, der immer wieder bemerkt, dass er sich neben Personen befinden muss, die er sonst nie seiner Gesellschaft gewürdigt hätte (Dostoevskij T. 8, 116). Rogožin fällt nach der ersten Begegnung mit ihr in eine Besessenheit (typisch bei

\footnotetext{
6 ,',(..) Das Gesicht eines zum Tode Verurteilten, eine Minute, bevor das Eisen niedersaust, während er noch auf dem Schafott steht und den Kopf gleich auf dieses Brett legen wird.' (...) ,....) Malen Sie das Schafott so, daß nur die letzte Stufe deutlich und ganz nahe zu sehen ist; der Verbrecher setzt den Fuß darauf: Der Kopf, das Gesicht weiß wie ein Blatt Papier, der Geistliche hält ihm das Kreuz hin, er sucht es gierig mit seinen blauen Lippen und schaut und - weiß alles. (...) “' $(93,97)$

7 „An Ihnen ist alles vollkommen... Sogar, daß Sie mager und blaß sind... man möchte Sie sich gar nicht anders vorstellen..." (203)
} 
Dostoevskij ist die Verbindung von psychischer Leidenschaft und physischem Fieber), die in eine Gehirnentzündung mündet; nach Nastas'ja Filippovnas Tod tritt seine langsame Genesung ein.

Nastas'ja Filippovna wird inszeniert und inszeniert sich selbst als schuldlos verführte, bereuende Frau à la Maria Magdalena, als schamlose Zuhälterin („эту бесстыжую“, 98), als eine reine Schönheit, die nicht von dieser Welt sein kann. Ihr Porträt wird von Fürst Myškin auch als orthodoxe Ikone, eine besondere Form der Abbildung, interpretiert, indem er das Abbild einer Frau küsst, die "furchtbar gelitten“ habe, und sich bereiterklärt, sie für immer zu verehren. Allerdings kann eine solche lkone kein lebendes Original besitzen, jede Heiligsprechung ist erst nach dem Tod des Abgebildeten möglich.

Die „Leiden“, die für Fürst Myškin eine besondere Schönheit darstellen, kommen dem Leser bekannt vor, stehen sie doch im Kontext literarischer Topoi, nach denen sowohl eine allzu verdorbene wie eine allzu gute Frau nur tot sein kann. „Вы книжная женщина“ $(472)^{8}$, wirft Aglaja ihrer Konkurrentin vor; Fürst Myškin entdeckt im Zimmer von Nastas'ja Filippovna, die „viele Poeme“ gelesen hat („женщина, прочитавшая много поэм“, ebd.), ein aufgeschlagenes Bibliotheksbuch, den französischen Roman "Madame Bovary", markiert sich die aufgeschlagene Seite und nimmt es mit auf die Suche nach dem verschollenen realen Objekt. Der Tod der Emma Bovary am Ende des Buches zeigt, wie eine Hure (die ihren Mann zudem in den Ruin getrieben hat) mit dem widerlichen Arsentod einer Ratte endet. Ein literarischer Text liefert einer Figur im anderen Text quasi eine Vorschau für deren baldigen Tod. Die Leichenblässe eines imaginierten Verurteilten, die unbeschriebenem „Papier“ ähnelt und gleichzeitig auf ein „Papier“, einen Richterspruch, zurückzuführen ist („стал вдруг белый как бумага, совершенно как белая писчая бумага“, 56), fällt mit der Blässe von Nastas'ja Fillipovna zusammen, die aus unterschiedlichen Perspektiven betont wird und deren Tod ein schriftlich fixiertes Vorbild hat; die Vollstreckung des Urteils ist nur eine Frage der Zeit.

Auch das Messer, mit dem Rogožin endlich zusticht, wird dafür benutzt, neue Bücher aufzuschneiden, und in einem Buch aufbewahrt. Ein aufgeschlitzter Körper gibt wie ein Buch einen Text frei, nämlich den ganzen Roman. Im Übrigen scheint der Tod ein Lieblingsthema der

8 Das Adjektiv „knižnaja“ kann sich sowohl auf die Belesenheit Nastas'ja Fillipovnas als auch auf ihre ,Unwirklichkeit', Fiktionalität aus der Sicht der anderen Figuren beziehen; S. Geier übersetzt es im Sinne einer Nachahmung, „Sie leben wie die Heldin eines Romans“ (823). 
Romanfiguren zu sein, vor allem von Fürst Myškin, der mit jedem seiner epileptischen Anfälle einen kleinen Tod und ein Ende aller Zeit erlebt („es wird keine Zeit mehr sein“, „времени больше не будет“, 189) den gleichen Ausdruck aus der Offenbarung des Johannes benutzt auch Ippolit bezüglich seines eigenen Todes (318).

Das Chaos, das Nastas'ja Filippovna anrichtet, wird mit ihrem Tod beglichen, eine Ordnung stellt sich wieder her. Unbestimmbares und Schreckliches sind nach E. Bronfen die kulturellen Attribute des Todes wie der Weiblichkeit, die von einem Mangel, dem Schrecken einer Kastration ${ }^{9}$ gekennzeichnet ist. Ähnlich wie das Gesicht des Verurteilten als künstlerisch produktives Motiv für ein Gemälde erscheint, wird Nastas'ja Filippovnas Leiche zum vollkommenden Kunstwerk, zum Höhepunkt der Gemäldegalerie im düsteren Haus Rogožins, das einem Friedhof ähnelt (338), und stellt einen Gegenentwurf zum HolbeinGemälde des toten Christus dar:

„Спавший был закрыт с головой белою простыней, но члены как-то неясно обозначались; видно только было, по возвышению, что лежит протянувшись человек. Кругом в беспорядке, на постели, в ногах, у самой кровати на креслах, на полу даже, разбросана была снятая одежда, богатое белое шелковое платье, цветы, ленты. На маленьком столике, у изголовья, блистали снятые и разбросанные бриллианты. В ногах сбиты были в комок какие-то кружева, и на белевших кружевах, выглядывая из-под простыни, обозначался кончик обнаженной ноги; он казался как бы выточенным из мрамора и ужасно был неподвижен.“ (503) 10

${ }^{9}$ Bronfen verschiebt das Zentrum der Kastrationsangst vom Phallus auf den „universellen, geschlechts-unspezifischen“ Ort des Nabels, der das erste Trauma, die erste Trennung markiert (82).

10 „Der Schlafende war bis über den Kopf mit einem weißen Laken zugedeckt, die Glieder jedoch hoben sich irgendwie undeutlich ab; man konnte nur an den Erhebungen erkennen, daß dort ein Mensch ausgestreckt lag. Ringsum in großer Unordnung, auf dem Bett, am Fußende, auf einem Sessel unmittelbar vor dem Bett, sogar auf dem Fußboden lagen hastig abgestreifte Kleidungsstücke, ein prachtvolles weißes Seidenkleid, Blumen, Bänder. Auf dem Tischchen am Kopfende funkelten abgelegte, achtlos hingeworfene Juwelen. Am Fußende bauschten sich zusammengeknüllte Spitzen, und inmitten des weißen Bausches sah unter dem Laken eine nackte Fußspitze hervor; sie schien wie aus Marmor gemeißelt und war furchtbar unbeweglich." (Dostojewskij 2016, 877) 
Rogožin bedeckt die Tote mit einer „guten, amerikanischen Wachsdecke“, außerdem mit einem Laken („хорошею, американскою клеенкой, а сверх клеенки уж простыней“), überlegt, sie mit Blumen zu schmücken, und präsentiert sie Fürst Myškin wie ein Ausstellungsstück in einer Galerie: „А она ведь как лежит... К утру, как посветлеет, посмотри." (504f.) $)^{11}$

Die Leiche führt das voyeuristische Betrachten weiter, ihre Schönheit übersteigt die der Fotografie und muss gänzlich verborgen werden, da sie sonst wahrscheinlich nicht auszuhalten wäre. Sie wird über Attribute (Kleid, Schmuck, Wäsche) fragmentiert, zerstückelt und als verdecktes Ganzes gleichzeitig idealisiert erfahren. Eine Leiche als Autoikone, als Doppelgänger (Bronfen 127), der sich selbst ähnelt, hat, im Unterschied zu einer Fotografie oder einem Gemälde, keine andere Referentialität mehr außer zu sich selbst. Es ist der Höhepunkt der Entfremdung zwischen Referent und Abbild - und gleichzeitig sind sie sich näher als nie zuvor.

Im Unterschied zur lebenden Nastas'ja Filippovna löst die Tote zunächst weder Angst noch Begehren aus, sodass sich eine Rückkehr ins Reale als unnötig erweist. Das Messer, das sie direkt ins Herz trifft („под самую левую грудь“, 505), ist ein endgültiger, triumphaler Sieg des Überlebenden über den Tod. Nastas'ja Filippovna wird zum gesicherten toten Körper, der „den Abstand von zwei Momenten der Unsicherheit“ ermöglicht, „von weiblicher Sexualität und Verfall“ (Bronfen 28). Die schöne Leiche kontrastiert mit dem verwesenden, hässlichen Leichnam Christi und produziert neue Zeichen; die weibliche Leiche ist eher dazu geeignet, den Betrachter von religiösen Zweifeln zu erlösen.

Einerseits kann die tote Frau endlich für sich gewonnen, allen Konkurrenten entrissen werden; ihre vollkommene Schönheit überspielt eine „totale() Destabilisierung von Kategorien wie Position, Ort und Bezug“" (Bronfen 80). Der Körper, der von Nastas'ja Filippova bleibt, beendet jegliche Unbestimmtheit, es tritt ein Stillstand ein („времени больше не будет“). Diese Schönheit kann aber ihren Mangel nicht langfristig verbergen; Rogožin beschließt zwar, sich nicht von der Leiche zu trennen („не отдавать“, 504), aber der einsetzende Verwesungsgeruch lässt sich nicht mit vier Flaschen antibakterieller Lösung („четыре стклянки ждановской жидкости“, 504) aufhalten. Rogožin macht Nastas'ja Filippovna fassbar, indem er sie tötet, bringt damit aber ein neues Artefakt zustande, das noch weniger greifbar, auf lange Sicht nicht konservierbar ist. Die Leiche ist ambivalent und

11 „Und wie sie daliegt... Gegen Morgen, wenn's hell wird, mußt du sie dir ansehen." (880) 
unheimlich, weil sie unbestimmt ist, sich weder in dieser noch in einer anderen Welt vollständig befindet, zudem die verdrängte Idee des eigenen Todes offen zur Schau stellt. Unheimlich ist aber auch die lebende Frau, die einen nötigen Gegensatz zum Mann und als immer präsente Erinnerung an seine Sterblichkeit zugleich seine Bedrohung verkörpert.

Bei der Wahl zwischen zwei Frauen entscheidet sich Fürst Myškin für Nastas'ja Filippovna, für den eigenen Verfall, und wird nach ihrem Tod wieder, diesmal endgültig, als „Idiot“ zurück in die Schweiz gefahren. Nastas'ja Fillipovna läuft kurz vor der Trauung dem Fürsten davon, der keine Frauen im sexuellen Sinne (14), dafür einen petite mort in seinen epileptischen Anfällen kennt, und flüchtet zurück zu Rogožin, der "die Todesmetapher der Liebe und des Beischlafs" (Macho 270) verwirklicht, indem er sie in der Hochzeitsnacht ersticht. Rogožin dagegen wird durch den Tod seiner Geliebten erlöst (vom Vermögen, das er verliert, ließe sie sich sowieso nicht kaufen) - im Gegensatz zu Fürst Myškins „Mitleid“ („жалость твоя, пожалуй, еще пуще моей любви“, Dostoevskij T. 8, 177) ${ }^{12}$ widersetzt sich die „Bosheit“ („,твою любовь от злости не отличишь“, ebd.) ${ }^{13}$ der Identifikation mit dem Objekt. Die Leiche Nastas'ja Filippovnas führt schließlich die beiden Männer zusammen, die eine Bruderschaft geschlossen haben und gegen ihren Willen zu Gegnern geworden sind; Rogožin besteht darauf, diese letzte Nacht gemeinsam zu verbringen und Wache an ihrer gemeinsamen toten Braut $z u$ halten ${ }^{14}$. Überhaupt erinnern alle Liebesverflechtungen im Roman an die These von Eve Sedgwick, der zufolge Frauen kulturelle Austauschobjekte seien und die eigentlichen, homosozialen Kontakte ermöglichten (Sedgwick 50f.).

Die Leiche Nastas'ja Filippovnas ist kein nackter, vergewaltigter Körper (wie etwa später Zinočka in Leonid Andreevs „Bezdna“ von $\left.1901^{15}\right)$, sondern bleibt ein in sich geschlossenes Rätsel. Alles ist sorgfältig verhüllt und bedeckt, nur die hervorschauende Spitze des Fußes verweist darauf, dass sich darunter ein Körper befindet; vielleicht gibt es auch keinen Körper mehr, vielleicht ist schon eine Auferstehung eingetreten. Im Gegensatz zu der Leiche auf Holbeins Gemälde, die ewig

12 „Am ehesten ist's so, daß dein Mitleid noch ärger ist als meine Liebe!“ (308)

13 "Nun, deine Liebe ist kaum von Haß zu unterscheiden (...). “ (Ebd.)

${ }^{14} \mathrm{Zu}$ den homoerotischen Konnotationen an dieser Stelle s. Hansen-Löve $49 \mathrm{f}$.

15 „(...) это голое женское тело, гладкое, упругое, как будто теплевшее под прикасающейся рукой“ (Andreev 365).

„(...) dieser nackte weibliche Körper, weich, nachgiebig, beinahe warm unter der Hand, die ihn berührte“ (Übers. S. K.). 
an der Wand hängen und ihre Wunden zur Schau stellen wird, löst die tote Nastas'ja Filippovna weder Ekel noch Mitleid aus. Sie scheint so fest zu schlafen, dass sie aus sicherer Entfernung unbemerkt betrachtet werden kann. Die beiden Betrachter haben einen Anderen vor sich, der zunächst tot genug ist, um ihnen das eigene Überleben zu bestätigen, und harmlos genug, um die Angst vor dem eigenen Verfall abzuwenden.

Der halbe Löffel Blut, der aus Nastas'ja Filippovna hervortritt, und die unsichtbare „innere Blutung“ („внутреннее излияние“, 505) wiederholen sich buchstäblich bei der "Sanften“:

„Какая она тоненькая в гробу, как заострился носик! Ресницы лежат стрелками. И ведь как упала - ничего не размозжила, не сломала! Только одна эта ,горстка крови'. Десертная ложка то есть. Внутренее сотрясение. Странная мысль: если бы можно было не хоронить? Потому что если ее унесут, то... о нет, унести почти невозможно!“ (Dostoevskij 1982, 35) ${ }^{16}$

Der ganze Text stellt den Monolog eines Ich-Erzählers, eines Pfandleihers, vor der Leiche seiner jungen Frau dar, die kurz davor aus dem Fenster gesprungen ist. Er versucht, sich an das kurze Eheleben mit der „Sanften“ zu erinnern, und einen Fehler in seinem kalkulierten Verhalten ihr gegenüber zu finden. Der Tod Nastas'ja Filippovnas ist beinahe ein Selbstmord, sie weiß um das für sie bereitgelegte Messer; der Selbstmord der "Sanften“ ist beinahe ein Mord, sie wird bei den Umerziehungsversuchen ihres Mannes vom Schweigen,erstickt'. In beiden Fällen geht es um die Schaffung eines Kunstwerkes, das Harmonie und Ganzheit vermittelt; die unversehrte Leiche schiebt die Frage nach dem Täter, der Verantwortung beiseite, vermittelt die Vorstellung eines schönen, friedlichen, natürlichen Todes, und braucht scheinbar nicht bestattet zu werden. Mehr noch, dem anonymen IchErzähler scheint es unmöglich, sich von der Leiche seiner Frau zu trennen: „...Вот пока она здесь-еще всё хорошо: подхожу и смотрю

\footnotetext{
16 „Wie schmal sie im Sarge aussieht, wie spitz ihr Näschen geworden ist! Die Wimpern liegen pfeilgerade, Und wie eigen sie hinuntergestürzt ist - nichts gebrochen, nichts zertrümmert! Nichts mehr als einzig die ,kleine Handvoll Blut'. Nämlich ein Dessertlöffel voll. Innere Erschütterung. Sonderbarer Gedanke: Wäre es vielleicht möglich, sie nicht zu beerdigen? Denn wenn man sie forttragen wird, so... o nein, es ist unmöglich, daß man sie forttragen wird! " (Dostojewskij 2015, 69f.)
} 
поминутно; а унесут завтра и - как же останусь один?“ (Dostoevskij $1982,6)^{17}$

Dieser Ich-Erzähler stellt sich ein Kollektiv von ironischen, zweifelnden, urteilenden Zuhörern vor, denen gegenüber er sich zu behaupten und Schuldgefühle abzuwehren versucht. Auch hier steht die "Sanfte" in einer Mittlerfunktion, sie soll ihrem Mann, der seinerzeit aus dem Offiziersdienst ausgetreten ist, die Rückkehr in seine alte Welt ermöglichen und ihn in den Augen der anderen Männer rehabilitieren. Die Stilisierung der Leiche verbindet, wie bei ihrer Vorgängerin, Schlaf und Tod als „Grenzerfahrungen“, die eine Abkehr von der Außenwelt, den Bruch mit jeder Sozialität (vgl. Macho 255f.) gemeinsam haben. Der metaphorische Schlaf schwächt den Tod der "Sanften“ als einen vorübergehenden Zustand $a b$, während sich die Leiche für immer jeglichen Machtansprüchen entzieht.

Anstelle einer Fotografie wird die "Sanfte“ von einer alten Ikone begleitet. Zusammen mit der Mutter Gottes („Богородица“, „Gottesgebärerin“, Dostoevskij 1982, 8) betritt sie die Wohnung des Pfandleihers, wird allmählich immer blasser („такая бледненькая“, „чрезвычайно бледна“, „страшно бледна“, 8, 20, 22) und springt mit ihr schließlich aus dem Fenster. Die Eigenschaften und Gedanken, die der Frau beharrlich vom Erzähler zugeschrieben werden, erinnern an ein tradiertes Heiligenbild, das, erneut, die Auslöschung des realen Objekts bedeutet.

Der Pfandleiher bedauert es, nicht mehr wahrgenommen zu werden: „Слепая, слепая! Мертвая, не слышит!“ (35) 18 . Dabei kann der Tod nur dann als ein „Sinnlichkeitsverlust“ bezeichnet werden, solange „ein potentielles Subjekt sinnlicher Wahrnehmung vorausgesetzt werden darf“ (Macho 253). Den Tod der "Sanften“ zu akzeptieren hieße, die eigene Bedeutsamkeit ihr gegenüber in Frage zu stellen. Gleichzeitig ermöglicht die anwesende Leiche erst die kurzzeitige Entfaltung als sprechendes und kreierendes Subjekt. Das Ausmaß der Erkenntnisse nimmt parallel zum langsam fortschreitenden Verfall des Leichnams zu, und im Gegensatz zu Fürst Myškin und Rogožin droht dem Ich-Erzähler weder eine Einlieferung ins Irrenhaus noch eine Verbannung nach Sibirien.

Auch die „Sanfte“ (ihr Namensersatz erinnert an eine typologisierte Rolle innerhalb eines Theaterstücks) beginnt kurz vor ihrem Tod zu lesen

\footnotetext{
17 „Solange sie noch hier ist - ist alles noch gut; ich kann mich ihr nähern und sie immer wieder anschauen; morgen aber, wenn man sie fortträgt - wie soll ich dann allein bleiben?" (6)

18 „Blind, blind! Tot, und kann mich nicht mehr hören!“ (70)
} 
und löst sich schließlich zu einem Kunstwerk und zum Medium fremder Zeichen auf. Im Vergleich zu Nastas'ja Filippovna, zu deren schon erwarteten Zersetzung, die das Auseinanderfallen des Sujets und das Ende des Romans bedeutet, weist die "Sanfte" stärker auf eine Wirklichkeit außerhalb ihrer selbst, auf eine reale Zeitlichkeit hin. Während Fürst Myškin in den Zustand eines „Idioten“ ohne Raum- und Zeitorientierung wechselt, für Rogožin ein neuer Lebensabschnitt, fünfzehn Jahre Lagerarbeit beginnen, spricht der Pfandleiher hektisch gegen ein Uhrpendel an (35), um eine Erzählung abzuschließen, solange die Leiche vor ihm liegt. Am Ende fehlen „fünf Minuten“ (32), um die "Sanfte" am Leben bzw. ihre Leiche als schön und unversehrt zu erhalten.

Der auf zwei Spieltischen unverhüllt zur Schau gelegte Körper (Karten finden sich auch neben seiner Vorgängerin, um deren Besitz ,gespielt' wird) erlaubt es dem Erzähler, sich an seine Geschichte in Form einer aufbauenden Rückwendung zu erinnern und an die Gegenwart anzuschließen, sodass das Ende der Erzählung an ihren Anfang, an die Leiche der "Sanften“ anschließen soll. Der zweite, erzählte Tod der „Sanften“, der ihren Sprung aus dem Fenster rekonstruiert, enthält eine Leerstelle, die keinen befriedigenden Übergang zur Präsenz des Leichnams gestalten lässt. Da der Ich-Erzähler zu dem Zeitpunkt außer Haus war, ist er an dieser entscheidenden Stelle gezwungen, auf die Erinnerung der alten Dienerin Luker'ja zurückzugreifen, und will sie als Zeugin (als Requisit der Leiche) ebenfalls für immer bei sich behalten: „(...) о, я теперь Лукерью ни за что не отпущу, она всё знает, она всю зиму была, она мне всё рассказывать будет“ $(32)^{19}$. In seinem absoluten Wissen über die "Sanfte“ ist er plötzlich vielen Menschen unterlegen, die den Sprung zufällig gesehen, sich um die Tote im Hof versammelt haben, und fällt, offenbar gekränkt, „mit aller Kraft“ über einen Mann aus der Menge her (33).

Der weibliche Körper wird diesmal nicht nur wie ein Buch aufgeschnitten, zu Ende gelesen und beiseitegelegt, sondern stellt den Anlass und die Bedingung für die ganze Erzählung dar. Bei dieser unheimlicheren, besitzergreifenden Totenwache ist alles, was von der "Sanften“ geblieben ist, nur über die Schilderung des Ich-Erzählers zugänglich (selbst Nastas'ja Filippovna kommt aus verschiedenen Perspektiven zu Wort). Gleichzeitig geht die Besetzung der Leiche erneut nicht ganz auf - es steht das Ende des potentiell unendlichen Sprechens (und Lebens) bevor, weil das phantastische, da unmögliche „Erzählen

19 ,"...) oh, Lukerja werde ich um nichts in der Welt gehen lassen, sie weiß alles, sie hat den ganzen Winter miterlebt, sie wird mir alles erzählen“ (64). 
des Endes" der Frau in jedem Fall auch das "Ende des Erzählens" (Hansen-Löve 39) des Mannes bedeutet. Der Ich-Erzähler fürchtet sich davor, die kostbare Leiche zu verlieren (das Wegbringen ersetzt euphemistisch die vollständige Verwesung), die wie ein Pfand jederzeit abgeholt werden kann, und beendet seinen Monolog erneut mit der Frage: „Нет, серьезно, когда ее завтра унесут, что ж я буду?“ (Dostoevskij 1982, 35) ${ }^{20}$

Während Fürst Myškin an der Photografie von Nastas'ja Filippovna das Porträt eines Verurteilten kurz vor der Hinrichtung erkennt und von dem „Wissen“ über das bevorstehende Ende fasziniert ist, geht der Pfandleiher weiter und versucht, das "Wissen“ der bereits toten "Sanften“ zu vereinnahmen. Der Unterschied zwischen einer stenografischen Schilderung der Gedanken eines Verurteilten und der Erzählung des Pfandleihers, die im Vorwort von Dostoevskij als „phantastisch“ parallelisiert werden (6), besteht darin, dass der eigene Tod nicht verbalisiert werden kann, die Darstellung eines fremden Todes zudem aber zwangsläufig in einer Instrumentalisierung und in einer Verteidigungsrede in eigener Sache mündet.

Die beiden Frauen, über die kaum etwas sicher bekannt ist, sind jeweils im Haus eines Mannes aufgebahrt, der sich um ihren Besitz bemüht und sie (wenn auch indirekt) ermordet hat. An die Stelle eines Messers tritt ein kompliziertes psychologisches System, mit dessen Hilfe der Ich-Erzähler die Umerziehung seiner Frau versuchte. Während die "Sanfte" als schweigendes Rätsel in erster Linie über den erzählenden Pfandleiher aussagt, bleibt Nastas'ja Filippovna in allen möglichen Perspektiven ein Rätsel, an dem auch ihre Idealisierung oder Verteufelung nichts ändert. Nachdem der vorausgeahnte Tod von Nastas'ja Filippovna eintritt und ihre Leiche von Außenstehenden entdeckt wird, verliert das weitere Erzählen jeden Sinn ${ }^{21}$; die IchErzählung des Pfandleihers, die den Tod der „Sanften“ wiederholen soll, scheitert an der narrativen Unmöglichkeit, den Tod zur Sprache zu bringen, und gleichzeitig über den Tod hinaus zu sprechen. Dabei bricht die Kommentierung und Umspielung auf der Metaebene aufgebautes Pathos immer wieder auf, sodass die Leichen wie die Texte eine Selbstreferentialität zur Schau stellen. Im Endeffekt ist jegliche Sinnzuschreibung in beiden Texten „nur über ihre Leiche“ möglich.

\footnotetext{
20 „Nein, im Ernst, wenn man sie morgen forttragen wird, was soll ich dann?“ (71) 21 Lediglich wird in einem kurzen Epilog grob das weitere Schicksal der Romanfiguren umrissen.
} 


\section{Literatur}

Andreev, Leonid. Sobranie sočinenij v šesti tomach. Tom 1. Bezdna. Moskva: Chudožestvennaja literatura, 1990.

Bachtin, Michail. Problemy poètiki Dostoevskogo. Moskva: Chudožestvennaja literatura, 1972.

Bronfen, Elisabeth. Nur über ihre Leiche. Tod, Weiblichkeit und Ästhetik. Deutsch von Thomes Lindquist. München: Kunstmann, 1994.

Dostoevskij, Fedor M. Polnoe sobranie sočinenij v 30. tomach. Tom 6. Prestuplenie i nakazanie. Leningrad: Nauka, 1973.

Dostoevskij, Fedor M. Polnoe sobranie sočinenij v 30. tomach. Tom 8. Idiot. Leningrad: Nauka, 1973.

Dostoevskij, Fedor M. Polnoe sobranie sočinenij v tridcati tomach. Tom 24. Dnevnik pisatelja za 1876 god. Krotkaja. Leningrad: Nauka, 1982.

Dostojewskij, Fjodor. Der Idiot. Neu übersetzt von Swetlana Geier. Frankfurt am Main: Fischer, 2016.

Dostojewskij, Fjodor. Die Sanfte. Aus dem Russischen übertragen von Johannes von Guenther. Ditzingen: Reclam, 2015.

Hansen-Löve, Aage A. "Grundzüge einer Thanatopoetik. Russische Beispiele von Puškin bis Čechov“. Wiener Slawistischer Alamanach. 60, 2007. 7-78.

Macho, Thomas H. Todesmetaphern. Frankfurt am Main: Suhrkamp 1987.

Sedgwick Kosofsky, Eve. Between Man. English Literature and Male Homosocial Desire. New York: Columbia University Press, 2016.

\section{THE FEMALE CORPSE IN DOSTOEVSKY}

The concept of the "beautiful female corpse" of E. Bronfen is used to analyse the narrative functions of the dead woman in Dostoevsky's "Idiot" ("The Idiot", 1868) and "Krotkaja" ("A Gentle Creature", 1876).

Keywords: femininity, death, corpse, Idiot, Nastas'ja Filippovna, Bronfen 